\title{
A LOWER BOUND FOR THE GENUS OF SELF-AMALGAMATION OF HEEGAARD SPLITTINGS
}

\author{
Fengling Li and Fengchun Lei*
}

\begin{abstract}
Let $M$ be a compact orientable closed 3-manifold, and $F$ a non-separating incompressible closed surface in $M$. Let $M^{\prime}=M-\eta(F)$, where $\eta(F)$ is an open regular neighborhood of $F$ in $M$. In the paper, we give a lower bound of genus of self-amalgamation of minimal Heegaard splitting $V^{\prime} \cup_{S^{\prime}} W^{\prime}$ of $M^{\prime}$ under some conditions on the distance of the Heegaard splitting.
\end{abstract}

\section{Introduction}

A Heegaard splitting of a 3-manifold $M$ is a decomposition $M=V \cup_{S} W$ of $M$ in which $V$ and $W$ are compression bodies such that $V \cap W=\partial_{+} V=\partial_{+} W=$ $S$ and $M=V \cup W . S$ is called a Heegaard surface of $M$. The genus $g(S)$ of $S$ is called the genus of the splitting $V \cup_{S} W$. We use $g(M)$ to denote the Heegaard genus of $M$, which is equal to the minimal genus of all Heegaard splittings of $M$. A Heegaard splitting $V \cup_{S} W$ for $M$ is minimal if $g(S)=g(M) . V \cup_{S} W$ is said to be weakly reducible if there are essential disks $D_{1} \subset V$ and $D_{2} \subset W$ with $\partial D_{1} \cap \partial D_{2}=\emptyset$. Otherwise, $V \cup_{S} W$ is strongly irreducible. Specially, let $M$ be a 3-manifold with boundary, and $\mathcal{F}$ a collection of boundary components of $M$. If $V \cup_{S} W$ is a Heegaard splitting of $M$ such that $\mathcal{F} \subset \partial_{-} V$ or $\mathcal{F} \subset \partial_{-} W$, then $M=V \cup_{S} W$ is called a Heegaard splitting relative to $\mathcal{F}$. In this case, if $g(S)$ is minimal among all the Heegaard splittings of $M$ relative to $\mathcal{F}$, then $g(S)$ is called the minimal genus of $M$ relative to $\mathcal{F}$, and is denoted by $g(M, \mathcal{F})$.

Let $M_{i}$ be a connected compact orientable 3-manifold, $F_{i}$ an incompressible boundary component of $M_{i}$ with $g\left(F_{i}\right) \geq 1, i=1,2$, and $F_{1} \cong F_{2}$. Let $\varphi: F_{1} \rightarrow F_{2}$ be a homeomorphism, and $M=M_{1} \cup_{\varphi} M_{2}$. Suppose $V_{i} \cup_{S_{i}} W_{i}$ is a Heegaard splitting of $M_{i}(i=1,2)$. Then $V_{1} \cup_{S_{1}} W_{1}$ and $V_{2} \cup_{S_{2}} W_{2}$ induce a natural Heegaard splitting $V \cup_{S} W$ of $M$ with $g(S)=g\left(S_{1}\right)+g\left(S_{2}\right)-g(F)$, which is called the amalgamation of $V_{1} \cup_{S_{1}} W_{1}$ and $V_{2} \cup_{S_{2}} W_{2}$ along $F_{1}$ and $F_{2}$. Thus we have that $g(M) \leq g\left(M_{1}\right)+g\left(M_{2}\right)-g(F)$.

Received May 11, 2009; Revised December 4, 2009.

2010 Mathematics Subject Classification. 57M99.

Key words and phrases. Heegaard distance, Heegaard genus, self-amalgamation.

* Supported in part by a key grant (No. 10931005) of NFSC. 
There exist examples which show that an amalgamation of two minimal genus Heegaard splittings of $M_{1}$ and $M_{2}$ is stabilized, see [1], [8] and [19] etc. On the other hand, it has been shown that under some conditions on the manifolds, the gluing maps, or the distances of the factor manifolds, the equality $g(M)=g\left(M_{1}\right)+g\left(M_{2}\right)-g(F)$ holds, see [9], [10], [20], [7] and [21] etc.

Suppose now that $F$ is a non-separating incompressible surface in $M$. Let $\eta(F)$ and $N(F)$ be the open and closed regular neighborhood of $F$ in $M$. We denote by $F_{1}$ and $F_{2}$ the two boundary components of $N(F)$. Let $M^{\prime}=$ $M-\eta(F)$ and $M^{\prime}=V^{\prime} \cup_{S^{\prime}} W^{\prime}$ be a Heegaard splitting relative to $\partial N(F)$. Then $M$ has a natural Heegaard splitting $V \cup_{S} W$ called the self-amalgamation of $V^{\prime} \cup_{S^{\prime}} W^{\prime}$ as follows:

Assume that $F_{1} \cup F_{2} \subset \partial_{-} W^{\prime}$ and let $\alpha_{i}$ be an unknotted arc in $W^{\prime}$ such that $\partial_{1} \alpha_{i} \subset \partial_{+} W^{\prime}$ and $\partial_{2} \alpha_{i} \subset F_{i}$ for $i=1,2$.

Let $\beta$ be an unknotted arc in $N(F)$ such that $\partial_{1} \beta=\partial_{2} \alpha_{1}$ and $\partial_{2} \beta=\partial_{2} \alpha_{2}$. Now let $N\left(\alpha_{1} \cup \beta \cup \alpha_{2}\right)$ be a closed regular neighborhood of $\alpha_{1} \cup \beta \cup \alpha_{2}$ in $W^{\prime} \cup N(F)$, and $\eta\left(\alpha_{1} \cup \beta \cup \alpha_{2}\right)$ be an open regular neighborhood of $\alpha_{1} \cup \beta \cup \alpha_{2}$ in $W^{\prime} \cup N(F)$. Let $V=V^{\prime} \cup N\left(\alpha_{1} \cup \beta \cup \alpha_{2}\right)$, and $W=W^{\prime} \cup N(F)-\eta\left(\alpha_{1} \cup\right.$ $\left.\beta \cup \alpha_{2}\right)$. Then $V \cup_{S} W$ is a Heegaard splitting of $M$. We call $V \cup_{S} W$ the self-amalgamation of $V^{\prime} \cup_{S^{\prime}} W^{\prime}$, and $M$ the self-amalgamation of $M^{\prime}$. It is clear $g(S)=g\left(S^{\prime}\right)+1$. Therefore, $g(M) \leq g\left(M^{\prime} ; \partial N(F)\right)+1$.

Qiu and Lei [13] and Du, Lei, and Ma [4] have given lower bounds of Heegaard genera of the self-amalgamation of 3-manifolds under some circumstances.

Theorem 1.1. Let $M$ be an orientable closed 3-manifold, and $F$ a nonseparating incompressible closed surface. Let $M^{\prime}=M-\eta(F)$. If $M^{\prime}$ has a Heegaard splitting $V^{\prime} \cup_{S^{\prime}} W^{\prime}$ with $d\left(S^{\prime}\right)>2 g\left(M^{\prime}\right)$, then $g(M) \geq g\left(M^{\prime}\right)-g(F)$. Furthermore, if $F$ is a torus, then $g(M) \geq g\left(M^{\prime}\right)+1$.

Theorem 1.2. Let $M$ be an orientable closed 3-manifold, and $F$ a nonseparating incompressible closed surface. Let $M^{\prime}=M-\eta(F)$. If $M^{\prime}$ has a Heegaard splitting $V^{\prime} \cup_{S^{\prime}} W^{\prime}$ relative to $\partial N(F)$ such that $d\left(S^{\prime}\right)>2\left(g\left(M^{\prime}, \partial N(F)\right)+\right.$ $2 g(F))$, then $M$ has a unique minimal Heegaard splitting up to isotopy, i.e., the self-amalgamation of $V^{\prime} \cup_{S^{\prime}} W^{\prime}$.

In this paper we give a lower bound for genera of self-amalgamations of Heegaard splittings under some condition on the distances of the Heegaard splittings as follows:

Theorem 1.3. Let $M$ be a compact orientable closed 3-manifold and $F$ a nonseparating incompressible closed surface in $M$. Let $M^{\prime}=M-\eta(F)$. Suppose $M^{\prime}$ has a Heegaard splitting $V^{\prime} \cup_{S^{\prime}} W^{\prime}$ with $d\left(S^{\prime}\right)>2(t+2 g(F))$, where $t$ is an integer with $1 \leq t \leq g\left(M^{\prime}\right)$. Then $g(M) \geq t+1$.

As a direct consequence of Theorem 1.3, we have: 
Corollary 1.4. Let $M$ be a compact orientable closed 3-manifold and $F$ a nonseparating incompressible closed surface in $M$. Let $M^{\prime}=M-\eta(F)$. Suppose $M^{\prime}$ has a Heegaard splitting $V^{\prime} \cup_{S^{\prime}} W^{\prime}$ with $d\left(S^{\prime}\right)>2\left(g\left(M^{\prime}\right)+2 g(F)\right)$. Then $g(M) \geq g\left(M^{\prime}\right)+1$. In particular, if $V^{\prime} \cup_{S^{\prime}} W^{\prime}$ is a Heegaard splitting relative to $\partial N(F)$, then the self-amalgamation of $V^{\prime} \cup_{S^{\prime}} W^{\prime}$ for $M$ is minimal.

In Section 2, we review some preliminaries which will be used in Section 3. The proof of Theorem 1.3 is given in Section 3 .

\section{Preliminaries}

In this section, we will review some fundamental facts on surfaces in 3manifolds.

Let $M$ be a 3-manifold. Suppose $F$ is a surface properly embedded in $M$. If $F$ is incompressible and not parallel to a sub-surface of $\partial M$, then $F$ is said to be an essential surface in $M$.

Let $M=V \cup_{S} W$ be a Heegaard splitting, $\alpha$ and $\beta$ be two essential simple closed curves in $S$. The distance $d(\alpha, \beta)$ of $\alpha$ and $\beta$ is the smallest integer $n \geq 0$ such that there is a sequence of essential simple closed curves $\alpha=$ $\alpha_{0}, \alpha_{1}, \ldots, \alpha_{n}=\beta$ in $S$ with $\alpha_{i-1} \cap \alpha_{i}=\emptyset$ for $1 \leq i \leq n$. The distance of the Heegaard splitting $V \cup_{S} W$ is defined to be $d(S)=\min \{d(\alpha, \beta)\}$, where $\alpha$ bounds an essential disk in $V$ and $\beta$ bounds an essential disk in $W . d(S)$ was first defined by Hempel in [6]. It is clear that $V \cup_{S} W$ is reducible if and only if $d(S)=0, V \cup_{S} W$ is weakly reducible if and only if $d(S) \leq 1$.

The following are some basic facts and results on Heegaard splittings.

Lemma 2.1 ([18]). Let $V$ be a compression body and $F$ an incompressible surface in $V$ with $\partial F \subset \partial_{+} V$. Then each component of $\overline{V-F}$ is a compression body.

Lemma $2.2([5])$. Let $V \cup_{S} W$ be a Heegaard splitting of $M$ and $F$ a properly embedded incompressible surface (possibly disconnected) in $M$. Then any component of $F$ is parallel to $\partial M$ or $d(S) \leq 2-\chi(F)$.

Let $M=V \cup_{S} W$ be a Heegaard splitting, and $F$ a boundary component of $M$. By gluing a $F \times I$ to $F$ and then amalgamating the standard Heegaard splitting of genus $2 g(F)$ of $F \times I$ (see [16]) with the given Heegaard splitting $(V, W)$ of $M$, we get a new Heegaard splitting of $M$. The construction above is called a boundary stabilization on the boundary component $F$. This was defined by Moriah in [11].

Lemma 2.3 ([17]). Suppose $P$ and $Q$ are Heegaard splitting surfaces for the compact orientable 3-manifold $M$. Then either $d(P) \leq 2 \operatorname{genus}(Q)$ or $Q$ is isotopic to $P$ or to a stabilization or boundary-stabilization of $P$.

Let $M=V \cup_{S} W$ be a strongly irreducible Heegaard splitting, and $\mathcal{F}$ a collection of essential surfaces in $M . \mathcal{F}$ is called a minimal separating system if 
$M-\mathcal{F}$ contains two components $M_{1}$ and $M_{2}$ and for any proper subset $\mathcal{F}^{\prime}$ of $\mathcal{F}, M-\mathcal{F}^{\prime}$ contains only one component. The following lemma is an extension of Schultens's lemma [18]. Bachman, Schleimer and Sedgwick [2] first proved Lemma 2.4 when $F$ is connected and closed.

Lemma 2.4 ([13]). Let $M=V \cup_{S} W$ be a strongly irreducible Heegaard splitting and $\mathcal{F}$ a minimal separating system in $M$ which cuts $M$ into two manifolds $M_{1}$ and $M_{2}$. Then $S$ can be isotoped such that

(1) each of $S \cap M_{1}$ and $S \cap M_{2}$ is incompressible; or

(2) one of $S \cap M_{1}$ and $S \cap M_{2}$, say $S \cap M_{1}$, is incompressible while all components of $S \cap M_{2}$ are incompressible except one bicompressible component; or

(3) one of $S \cap M_{1}$ and $S \cap M_{2}$, say $S \cap M_{1}$, is incompressible while $S \cap M_{2}$ is compressible. Furthermore, there is a Heegaard surface $S^{\prime}$ isotopic to $S$ such that

(i) at most one component of $S^{\prime} \cap M_{1}$ is compressible while $S^{\prime} \cap M_{2}$ is incompressible, and

(ii) $S^{\prime}$ is obtained by $\partial$-compressing $S$ in $M_{2}$ only one time.

Proof. Let $\left\{H_{1}, H_{2}\right\}=\{W, V\}$. If each component of $S \cap M_{1}$ and $S \cap M_{2}$ is incompressible, then Lemma 2.4(1) holds. If one of $S \cap M_{1}$ and $S \cap M_{2}$ is bicompressible, then, since $V \cup_{S} W$ is strongly irreducible, Lemma 2.4(2) holds. We may assume that

Assumption (1) one or both of $S \cap M_{1}$ and $S \cap M_{2}$ are compressible in $M_{1} \cap H_{1}$ and $M_{2} \cap H_{1}$, respectively.

Assumption (2) $S \cap M_{i}$ is incompressible in $M_{i} \cap H_{2}$ for $i=1,2$.

Since $\mathcal{F}$ is a collection of essential surfaces in $M, H_{1}$ and $H_{2}$ are non-trivial compression bodies. Let $D$ be an essential disk of $H_{2}$ such that $|D \cap \mathcal{F}|$ is minimal among all essential disks in $H_{2}$. By Assumption (2), $|D \cap \mathcal{F}|>0$. Furthermore, we may assume that

Assumption (3) $S$ is a strongly irreducible Heegaard surface such that $|D \cap \mathcal{F}|$ is minimal among all Heegaard surfaces isotopic to $S$ and satisfying Assumptions (1) and (2).

Let $a$ be an outermost component of $D \cap \mathcal{F}$ on $D$. This means that $a$, together with an arc $b$ on $\partial D(\subset S)$, bounds a disk $B$ in $D$ which lies in either $M_{1} \cap H_{2}$ or $M_{2} \cap H_{2}$ such that $B \cap \mathcal{F}=a$, and we may assume that $B \subset M_{2} \cap H_{2}$. By the minimality of $|D \cap \mathcal{F}|, B$ is a $\partial$-compressing disk of $S \cap M_{2}$.

Now there are two cases:

Case 1. $S \cap M_{1}$ is compressible in $M_{1} \cap H_{1}$ ( $S \cap M_{2}$ is compressible or incompressible in $\left.M_{2} \cap H_{1}\right)$.

Now let $S^{\prime}$ be the Heegaard surface of $M$ obtained by $\partial$-compressing $S$ along $B$. In fact, $S^{\prime}$ is isotopic to $S$. We denote by $H_{1}^{\prime}$ and $H_{2}^{\prime}$ the two components of $M-S^{\prime}$. We may assume that $H_{1} \subset H_{1}^{\prime}$. Since the $\partial$-compression is done in $M_{2} \cap H_{2}, M_{1} \cap H_{1} \subset M_{1} \cap H_{1}^{\prime}$ and $S \cap M_{1} \subset S^{\prime} \cap M_{1}$. Since $S \cap M_{1}$ is 
compressible in $M_{1} \cap H_{1}, S^{\prime} \cap M_{1}$ is compressible in $M_{1} \cap H_{1}^{\prime}$. Now if $S^{\prime} \cap M_{1}$ is compressible in $M_{1} \cap H_{2}^{\prime}$, then Lemma 2.4(2) holds.

Suppose that $S^{\prime} \cap M_{1}$ is incompressible in $M_{1} \cap H_{2}^{\prime}$. If $S^{\prime} \cap M_{2}$ is compressible $M_{2} \cap H_{2}^{\prime}$, this contradicts Assumption (2). Hence $S^{\prime} \cap M_{2}$ is incompressible in $M_{2} \cap H_{2}^{\prime}$. Now $D \cap H_{2}^{\prime}$ is an essential disk in $H_{2}^{\prime}$. But $\left|D \cap H_{2}^{\prime} \cap \mathcal{F}\right|=$ $|D \cap \mathcal{F}|-1$. This contradicts Assumption (3) regardless of compressibility or incompressibility of $S^{\prime} \cap M_{2}$ in $M_{2} \cap H_{1}^{\prime}$.

Case 2. $S \cap M_{2}$ is compressible in $M_{2} \cap H_{1}$, and $S \cap M_{1}$ is incompressible in $M_{1} \cap H_{1}$.

Similarly, let $S^{\prime}$ be the Heegaard surface of $M$ obtained by $\partial$-compressing $S$ along $B$. We denote by $H_{1}^{\prime}$ and $H_{2}^{\prime}$ the two components of $M-S^{\prime}$. We may assume that $H_{1} \subset H_{1}^{\prime}$. Since the $\partial$-compression is done in $M_{2} \cap H_{2}$, $S \cap M_{1} \subset S^{\prime} \cap M_{1}$. By observation we can see that $S^{\prime} \cap M_{1}$ is incompressible in $M_{1} \cap H_{1}^{\prime}$ since new component of $S^{\prime} \cap M_{1}$ is obtained by attaching a band and new component of $M_{1} \cap H_{1}^{\prime}$ is obtained by attaching a 1-handle incident to the band. At most one component of $S^{\prime} \cap M_{1}$ is compressible in $M_{1} \cap H_{2}^{\prime}$ since $S \cap M_{1}$ is incompressible in $M_{1} \cap H_{2}$ by Assumption (2).

Now if $S^{\prime} \cap M_{2}$ is compressible $M_{2} \cap H_{2}^{\prime}$, this contradicts Assumption (2). Hence $S^{\prime} \cap M_{2}$ is incompressible $M_{2} \cap H_{2}^{\prime}$. If $S^{\prime} \cap M_{2}$ is incompressible in $M_{2} \cap H_{1}^{\prime}$, then Lemma 2.4(3) holds.

Suppose that $S^{\prime} \cap M_{2}$ is compressible in $M_{2} \cap H_{1}^{\prime}$. If it is the case that one component of $S^{\prime} \cap M_{1}$ is compressible in $M_{1} \cap H_{2}^{\prime}$, this contradicts the strong irreducibility of $S^{\prime}$. Hence the remaining case is that $S^{\prime} \cap M_{1}$ is incompressible in $M_{1} \cap H_{2}^{\prime}$, while $\left|D \cap H_{2}^{\prime} \cap \mathcal{F}\right|=|D \cap \mathcal{F}|-1$. This contradicts Assumption (3).

Lemma 2.5 ([4]). Let $S, S_{1}, S_{2}$ be three Heegaard surfaces of $M$ such that $S_{1} \cap S_{2}=\emptyset$ and the component of $M-S_{1} \cup S_{2}$ containing $S_{1}$ and $S_{2}$ contains at least one component of $\partial M$. Then at least one $S_{i}$ is not obtained by doing stabilizations on $S$.

Proof. Suppose both $S_{1}$ and $S_{2}$ are obtained by doing stabilizations on $S$.

Since each Heegaard surface separates $M, S_{1}$ and $S_{2}$ are disjoint, $M-S_{1} \cup$ $S_{2}$ has three components $M_{1}, M_{*}, M_{2}, M=M_{1} \cup_{S_{1}} M_{*} \cup_{S_{2}} M_{2}$. By the assumption, we have $\partial M_{*}=S_{1} \cup S_{2} \cup S_{*}$, where $S_{*}$ is a non-empty union of components of $\partial M$.

Suppose $S^{\prime}$ is a stabilization of $S$. We describe $S^{\prime}$ in a slightly different way. Let $N(S)$ be a closed regular neighborhood of $S$ in $M$. Identify a suitable component of $N(S)-S$ with $S \times[0,1]$ so that $S=S \times\{0\}$. Then $S^{\prime}=\partial(S \times$ $[0,1] \cup N(\alpha))-S$, where $\alpha$ is an arc in $M$ with $\alpha \cap S \times[0,1]=\partial \alpha \subset S \times\{1\}$ and $N(\alpha)$ is a 1 -handle attached to $S \times[0,1]$. Now the 3-manifold $S \times[0,1] \cup N(\alpha)$ provides a homology from $S$ to $S^{\prime}$, and moreover this homology is carried in a regular neighborhood of a 2-complex $S \times\{1\} \cup \alpha$ in $M$. 
Since $S_{i}$ is obtained by a sequence of stabilizations of $S$, by induction we have that $S$ and $S_{i}$ are homological, and moreover the homology is carried in a regular neighborhood of a 2-complex in $M$. Hence $S_{1}$ and $S_{2}$ are homological in $M$ and the homology is carried in a regular neighborhood $N(X)$ of a 2-complex $X$ in $M, S_{1}, S_{2} \subset X$.

Claim. Either $\partial M_{1} \neq S_{1}$ or $M_{1}$ is not a subset of $N(X)$. The similar is true for $M_{2}$.

Proof of Claim. We are going to prove the claim by contradiction. Suppose $\partial M_{1}=S_{1}$ and $M_{1} \subset N(X)$.

Note $N(X) \cap M_{1}$ is a regular neighborhood of $X \cap M_{1}$ in $M_{1}$. Let $D\left(M_{1}\right)$ be the double of $M_{1}$, which is obtained by gluing two copies of $M_{1}$ along their boundaries via the identity. Let $D\left(X \cap M_{1}\right)$ (resp. $\left.D\left(N(X) \cap M_{1}\right)\right)$ be the union of two copies of $X \cap M_{1}$ (resp. $\left.N(X) \cap M_{1}\right)$ in $D\left(M_{1}\right)$. Then $D\left(N(X) \cap M_{1}\right)$ is a regular neighborhood of $D\left(X \cap M_{1}\right)$ in $D\left(M_{1}\right)$.

Now $D\left(M_{1}\right)$ is a closed 3-manifold and $D\left(N(X) \cap M_{1}\right)=D\left(M_{1}\right)$. This is not possible, since $D\left(N(X) \cap M_{1}\right)$ has the 2-complex $D\left(X \cap M_{1}\right)$ as a deformation retract, which cannot be a closed 3 -manifold. So the claim is proved.

Let $M_{i}^{\prime}=M_{i}$ if $\partial M_{i} \neq S_{i}$, and otherwise $M_{i}^{\prime}=M_{i}-B_{i}^{3}$ where $B_{i}^{3} \subset \operatorname{int} M_{i}$ is a small 3-ball disjoint from $N(X)$. Let $M^{\prime}=M-B_{1}^{3}-B_{2}^{3}$. Clearly

(1) $N(X) \subset M^{\prime}$,

(2) $M^{\prime}=M_{1}^{\prime} \cup_{S_{1}} M_{*} \cup_{S_{2}} M_{2}^{\prime}, \partial M_{*}=S_{1} \cup S_{2} \cup S_{*}, \partial M_{1}^{\prime}=S_{1} \cup S_{1}^{\prime}, \partial M_{2}^{\prime}=$ $S_{2} \cup S_{2}^{\prime}$. Each one of $S_{*}, S_{1}^{\prime}, S_{2}^{\prime}$ is non-empty.

Since $N(X)$ carries the homology from $S_{1}$ to $S_{2}, S_{1}$ and $S_{2}$ are still homologous in $M^{\prime}$.

On the other hand, $S_{1}$ and $S_{2}$ are two closed disjoint orientable surfaces in orientable 3-manifold $M^{\prime}, S_{1}$ and $S_{2}$ are homological in $M^{\prime}$ if and only if $S_{1} \cup S_{2}$ cobounds a submanifold in $M^{\prime}$ or each of $S_{1}$ and $S_{2}$ bounds a submanifold and homologically trivial, which is not possible by (2).

\section{The proof of the main theorem}

Now we come to the proof of Theorem 1.3.

Proof of Theorem 1.3. By assumption, $M^{\prime}=M-\eta(F)$, and $V^{\prime} \cup_{S^{\prime}} W^{\prime}$ is a Heegaard splitting of $M^{\prime}$ with $d\left(S^{\prime}\right)>2(t+2 g(F))>0$, then by Haken's lemma (refer to [3]), $M^{\prime}$ and $M$ are irreducible.

Suppose that the inequality $g(M) \geq t+1$ does not hold, then there exists a minimal Heegaard splitting $V \cup_{S} W$ of $M$ with $g(S)<t+1$.

We divide it into the following two cases to discuss.

Case 1. The Heegaard splitting $V \cup_{S} W$ is strongly irreducible.

Claim 1. $S$ can be isotoped so that $S \cap M^{\prime}$ is bicompressible while $S \cap N(F)$ is incompressible. 
Proof of Claim 1. In this case, $\partial N(F)$ is a minimal separating system in $M$ which cuts $M$ into $M^{\prime}$ and $N(F)$. By Lemma 2.4, $S$ can be isotoped to one of the following three cases:

(1) $S \cap M^{\prime}$ and $S \cap N(F)$ are incompressible.

Since $g(S)<t+1$ and $d\left(S^{\prime}\right)>2(t+2 g(F))$, by Lemma $2.2, S \cap M^{\prime}$ is $\partial$-parallel in $M^{\prime}$, then $S$ is isotopic to $F$, a contradiction.

(2) one of $S \cap M^{\prime}$ and $S \cap N(F)$ is bicompressible while the other is incompressible.

By the arguments in (1), $S \cap M^{\prime}$ is bicompressible while $S \cap N(F)$ is incompressible.

(3) $S \cap M^{\prime}$ is compressible while $S \cap N(F)$ is incompressible. Furthermore, there is a Heegaard surface $S^{*}$ isotopic to $S$ such that $S^{*} \cap M^{\prime}$ is incompressible and at most one component of $S^{*} \cap N(F)$ is compressible. By the same arguments as (1), this is impossible. This completes the proof of Claim 1.

By Claim 1, we may assume that $S \cap M^{\prime}$ is bicompressible while $S \cap N(F)$ is incompressible. Furthermore, we assume that $|S \cap N(F)|$ is minimal among all Heegaard surfaces isotopic to $S$ and satisfying the above conditions.

Since $V \cup_{S} W$ is strongly irreducible, there is only one component, say $P$, of $S \cap M^{\prime}$ which is bicompressible. And any other component of $S \cap M^{\prime}$ is incompressible. Suppose that there is a component of $S \cap M^{\prime}$ besides $P$, say $Q$, which is incompressible, then by Lemma $2.2, Q$ is $\partial$-parallel in $M^{\prime}$, then $Q$ can be isotoped to be disjoint from $M^{\prime}$. This contradicts the minimality of $|S \cap N(F)|$. Thus $S \cap M^{\prime}$ has only one component, it is connected.

Obviously, any component of $\partial N(F) \cap V$ is incompressible in $V$, and any component of $\partial N(F) \cap W$ is incompressible in $W$. Then by Lemma 2.1, any component of $V \cap M^{\prime}$ and $W \cap M^{\prime}$ is a compression body. Since $S \cap M^{\prime}$ is connected, $V \cap M^{\prime}$ is one compression body, and so is $W \cap M^{\prime}$.

By the above arguments, $S \cap M^{\prime}$ is connected and bicompressible. Let $S_{V}$ be the surface obtained by maximally compressing $S \cap M^{\prime}$ in $V \cap M^{\prime}$. We may assume that $S \cap M^{\prime}$ is compressed to $S_{V}$ in $V \cap M^{\prime}$ by cutting $S \cap M^{\prime}$ open along a collection $\mathcal{D}=\left\{D_{1}, \ldots, D_{n}\right\}$ of pairwise disjoint compressing disks in $V \cap M^{\prime}$. Since $V \cup_{S} W$ is strongly irreducible, by the No nesting Lemma [14], $S_{V}$ is incompressible in $M^{\prime}$. Then by Lemma 2.2, we know that any component of $S_{V}$ is $\partial$-parallel in $M^{\prime}$.

Let $A_{1}, \ldots, A_{r}$ be all the components of $S_{V}$ with boundary, $\partial A_{i} \subset \partial N(F)$ for $1 \leq i \leq r$. Suppose that each $A_{i}$ is parallel to a subsurface $A_{i}^{\prime}$ of $\partial N(F)$ for $1 \leq i \leq r$.

Claim 2. For any components $A_{i}, A_{j}$ of $S_{V}, A_{i}^{\prime} \cap A_{j}^{\prime}=\emptyset$.

Proof of Claim 2. Suppose that there are two components of $S_{V}$, say $A_{i_{0}}$ and $A_{j_{0}}$, such that $A_{i_{0}}^{\prime} \cap A_{j_{0}}^{\prime} \neq \emptyset$, we may further assume that $A_{i_{0}}^{\prime} \subset A_{j_{0}}^{\prime}$. Then set $\mathcal{A}_{1}=\left\{A_{i}: A_{i}^{\prime} \subset A_{j_{0}}^{\prime}, 1 \leq i \leq r, i \neq j_{0}\right\}$ and $\mathcal{A}_{2}=\left\{A_{i}: A_{i}^{\prime} \cap A_{j_{0}}^{\prime}=\emptyset, 1 \leq i \leq r\right\}$. We claim that $\mathcal{A}_{2}=\emptyset$. Otherwise, since $S \cap M^{\prime}$ is connected, there must exist 
$A_{i_{1}} \in \mathcal{A}_{1}, A_{i_{2}} \in \mathcal{A}_{2}$, and $D_{p_{1}}, D_{p_{2}} \in \mathcal{D}$ such that $D_{p_{1}} \cap D_{p_{2}}=\emptyset$ and in the compression, the two copies of $D_{p_{k}}$ lie in $A_{i_{k}}$ and $A_{j_{0}}$ respectively, $k=1,2$. But this contradicts to the assumption that $S \cap M^{\prime}$ is separating in $M^{\prime}$. Thus $\mathcal{A}_{2}=\emptyset$. We denote by $W_{A_{j_{0}}}$ the handlebody bounded by $A_{j_{0}}$ and $A_{j_{0}}^{\prime}$ in $M^{\prime}$. Then all components of $S_{V}$ lie in $W_{A_{j_{0}}}$, so $S$ can be isotoped to be disjoint from $M^{\prime}$ in $M$, a contradiction. This completes the proof of Claim 2.

By Claim 2, for each component of $\partial N(F) \cap V$, say $A_{i_{*}}^{\prime}$, there is one and only one component $A_{i_{*}}$ of $S_{V}$ which is parallel to $A_{i_{*}}^{\prime}$. Let $B_{1}, \ldots, B_{t}$ be the components of $\overline{\partial N(F)-\cup_{i=1}^{r} A_{i}^{\prime}}=\partial N(F) \cap W$. Take a small regular neighborhood $B_{i} \times I$ of $B_{i}$ in $W \cap M^{\prime}$, where $B_{i} \times\{0\}=B_{i}, i=1,2, \ldots, t$. Set $V_{1}^{\prime}=\left(V \cap M^{\prime}\right) \bigcup \cup_{i=1}^{t} B_{i} \times I$ and $W_{1}^{\prime}=\overline{M^{\prime}-V_{1}^{\prime}}$. Then $V_{1}^{\prime}$ is obtained from $\partial N(F) \times I$ by adding 1-handles whose co-cores are disks in $\mathcal{D}$, so $V_{1}^{\prime}$ is a compression body. Note that $W_{1}^{\prime}=\overline{\left(W \cap M^{\prime}\right)-\cup_{i=1}^{t} B_{i} \times I} \cong W \cap M^{\prime}, W_{1}^{\prime}$ is a compression body. Let $S_{1}^{\prime}=\partial_{+} V_{1}^{\prime}$. Then it is obvious that $S_{1}^{\prime}=\partial_{+} W_{1}^{\prime}$. Thus, $S_{1}^{\prime}$ is a Heegaard surface of $M^{\prime}$. Since $S \cap M^{\prime}$ is compressible in $W \cap M^{\prime}$, there exists a compressing disk $D$ of $S \cap M^{\prime}$ in $W \cap M^{\prime}$ with $D \cap\left(B_{i} \times I\right)=\emptyset$ and $D \subset W_{1}^{\prime}$. Since $\partial B_{i} \times I$ are spanning annuli in $V_{1}^{\prime}$, there exists an essential disk $E$ in $V_{1}^{\prime}$ with $E \cap\left(\partial B_{i} \times I\right)=\emptyset$ (cf. [12] Lemma 2.1). Thus $d\left(S_{1}^{\prime}\right) \leq 2$. Let $S_{1}=\left(S \cap M^{\prime}\right) \cup(\partial N(F) \cap W)$. Then $S_{1}^{\prime}$ is the surface obtained from $S_{1}$ by pushing $\partial N(F) \cap W$ slightly into $W \cap M^{\prime}$.

Now let $S_{2}=\left(S \cap M^{\prime}\right) \cup(\partial N(F) \cap V)$, we denote by $S_{2}^{\prime}$ the surface obtained from $S_{2}$ by pushing $\partial N(F) \cap V$ slightly into $M^{\prime} \cap V$. By similar arguments as above, we know that $S_{2}^{\prime}$ is also a Heegaard surface of $M^{\prime}$ and $d\left(S_{2}^{\prime}\right) \leq 2$. By a small isotopy of $S_{1}^{\prime}$, we may assume that $S_{1}^{\prime} \cap S_{2}^{\prime}=\emptyset$. By the construction of $S_{1}^{\prime}$ and $S_{2}^{\prime}$, we know that the component of $M^{\prime}-S_{1}^{\prime} \cup S_{2}^{\prime}$ containing $S_{1}^{\prime}$ and $S_{2}^{\prime}$ also contains $\partial N(F)$.

Since $\chi\left(S \cap M^{\prime}\right) \geq \chi(S)>-2 t$, and

$$
\begin{aligned}
\chi\left(S_{1}^{\prime}\right) & =\chi\left(S \cap M^{\prime}\right)+\chi(\partial N(F) \cap W) \\
& \geq \chi(S)+\chi(\partial N(F)) \\
& =\chi(S)+2 \chi(F),
\end{aligned}
$$

we have

$$
g\left(S_{1}^{\prime}\right)<t+2 g(F)-1
$$

Similarly,

$$
g\left(S_{2}^{\prime}\right)<t+2 g(F)-1 .
$$

Since $d\left(S^{\prime}\right)>2(t+2 g(F))>2 g\left(S_{i}^{\prime}\right) \geq 2 g\left(M^{\prime}\right)$, by Lemma $2.3, V^{\prime} \cup_{S^{\prime}} W^{\prime}$ is the unique minimal Heegaard splitting of $M^{\prime}$ up to isotopy, and $S_{i}^{\prime}$ is isotopic to $S^{\prime}$ or to a possible stabilization or boundary-stabilization of $S^{\prime}$ for $i=1,2$. But $d\left(S^{\prime}\right)>2(t+2 g(F))>2$ while $d\left(S_{i}^{\prime}\right) \leq 2, S_{i}^{\prime}$ cannot be isotopic to the 
unique minimal Heegaard surface $S^{\prime}$ of $M^{\prime}$ for $i=1,2$. Hence $S_{i}^{\prime}$ is obtained by doing stabilization or boundary-stabilization on $S^{\prime}$ for $i=1,2$.

There are two subcases:

Subcase 1. $M^{\prime}=V^{\prime} \cup_{S^{\prime}} W^{\prime}$ is a Heegaard splitting relative to $\partial N(F)$.

By Lemma 2.5, one of $S_{1}^{\prime}$ and $S_{2}^{\prime}$, say $S_{1}^{\prime}$, is obtained by doing boundarystabilizations on $S^{\prime}$ at least one time. Since $S_{1}^{\prime}$ and $S^{\prime}$ are both Heegaard splittings relative to $\partial N(F), S_{1}^{\prime}$ is obtained by doing boundary-stabilizations on $S^{\prime}$ at least two times. Hence $g\left(S_{1}^{\prime}\right) \geq g\left(M^{\prime}\right)+2 g(F)$, a contradiction.

Subcase 2. $M^{\prime}=V^{\prime} \cup_{S^{\prime}} W^{\prime}$ is a Heegaard splitting with $F_{1} \subset \partial_{-} V^{\prime}$ and $F_{2} \subset \partial_{-} W^{\prime}$.

Since $F_{1} \subset \partial_{-} V^{\prime}$ and $F_{2} \subset \partial_{-} W^{\prime}$, and by the construction of $S_{1}^{\prime}$ and $S_{2}^{\prime}, S_{1}^{\prime}$ and $S_{2}^{\prime}$ are Heegaard surfaces relative to $\partial N(F)$. By Lemma 2.3 and (1), $S_{1}^{\prime}$ is obtained from $S^{\prime}$ by doing boundary-stabilizations at least one time, hence $g\left(S_{1}^{\prime}\right) \geq g\left(S^{\prime}\right)+g(F)$. By the similar arguments, we have $g\left(S_{2}^{\prime}\right) \geq g\left(S^{\prime}\right)+g(F)$.

Now

$$
\chi\left(S_{1}^{\prime}\right)=\chi\left(S \cap M^{\prime}\right)+\chi(\partial N(F) \cap W) \leq 2-2\left(g\left(M^{\prime}\right)+g(F)\right),
$$

and

$$
\chi\left(S_{2}^{\prime}\right)=\chi\left(S \cap M^{\prime}\right)+\chi(\partial N(F) \cap V) \leq 2-2\left(g\left(M^{\prime}\right)+g(F)\right),
$$

hence

$$
\begin{aligned}
2 \chi\left(S \cap M^{\prime}\right) & \leq 4-4\left(g\left(M^{\prime}\right)+g(F)\right)-\chi(\partial N(F) \cap W)-\chi(\partial N(F) \cap V) \\
& =4-4\left(g\left(M^{\prime}\right)+g(F)\right)-2 \chi(F),
\end{aligned}
$$

then $\chi\left(S \cap M^{\prime}\right) \leq-2 g\left(M^{\prime}\right)$, and $\chi(S \cap N(F)) \leq 0$, now we have $g(S) \geq$ $g\left(M^{\prime}\right)+1 \geq t+1$, a contradiction to our assumption.

Case 2. The Heegaard splitting $V \cup_{S} W$ is weakly reducible.

Now $M=V \cup_{S} W$ is irreducible and weakly reducible, then $V \cup_{S} W$ has an untelescoping [15] as

$$
V \cup_{S} W=\left(V_{1} \cup_{S_{1}} W_{1}\right) \cup_{H_{1}} \ldots \cup_{H_{n-1}}\left(V_{n} \cup_{S_{n}} W_{n}\right)
$$

where $n \geq 2$, each component of $H_{1}, \ldots, H_{n-1}$ is an incompressible closed surface in $M$, and $M_{i}=V_{i} \cup_{S_{i}} W_{i}$ is a non-trivial strongly irreducible Heegaard splitting for $1 \leq i \leq n$. Since $V \cup_{S} W$ is minimal, $g(S)=g(M)<t+1$. Note that $g\left(H_{i}\right)<g(S)$. Since $d\left(S^{\prime}\right)>2(t+2 g(F))$, by Lemma 2.2 , any component of $H_{i} \cap M^{\prime}$ is $\partial$-parallel in $M^{\prime}$ for each $i$, then $H_{i}$ can be isotoped to be disjoint from $M^{\prime}$ for each $i$. This means that each component of $H_{1}, \ldots, H_{n-1}$ is parallel to $F$. Now one of the manifolds $M_{1}, \ldots, M_{n}$ is homeomorphic to $M^{\prime}$, and each of the other is homeomorphic to $F \times I$.

Suppose some $M_{i_{0}}$ is homeomorphic to $M^{\prime}, V_{i_{0}} \cup_{S_{i_{0}}} W_{i_{0}}$ is a Heegaard splitting of $M^{\prime}$, we have $g\left(S_{i_{0}}\right) \leq g(S)-1<t \leq g\left(M^{\prime}\right)$, a contradiction. This case cannot happen.

This completes the proof of Theorem 1.3. 


\section{References}

[1] D. Bachman and R. Derby-Talbot, Degeneration of Heegaard genus, a survey, Workshop on Heegaard Splittings, 1-15, Geom. Topol. Monogr., 12, Geom. Topol. Publ., Coventry, 2007.

[2] D. Bachman, S. Schleimer, and E. Sedgwick, Sweepouts of amalgamated 3-manifolds, Algebr. Geom. Topol. 6 (2006), 171-194.

[3] A. J. Casson and C. McA Gordon, Reducing Heegaard splittings, Topology Appl. 27 (1987), no. 3, 275-283.

[4] K. Du, F. Lei, and J. Ma, Distance and self-amalgamation of Heegaard splittings, preprint.

[5] K. Hartshorn, Heegaard splittings of Haken manifolds have bounded distance, Pacific J. Math. 204 (2002), no. 1, 61-75.

[6] J. Hempel, 3-manifolds as viewed from the curve complex, Topology 40 (2001), no. 3, 631-657.

[7] T. Kobayashi and R. Qiu, The amalgamation of high distance Heegaard splittings is always efficient, Math. Ann. 341 (2008), no. 3, 707-715.

[8] T. Kobayashi, R. Qiu, Y. Rieck, and S. Wang, Separating incompressible surfaces and stabilizations of Heegaard splittings, Math. Proc. Cambridge Philos. Soc. 137 (2004), no. 3, 633-643.

[9] M. Lackenby, The Heegaard genus of amalgamated 3-manifolds, Geom. Dedicata 109 (2004), 139-145.

[10] T. Li, On the Heegaard splittings of amalgamated 3-manifolds, Workshop on Heegaard Splittings, 157-190, Geom. Topol. Monogr., 12, Geom. Topol. Publ., Coventry, 2007.

[11] Y. Moriah, On boundary primitive manifolds and a theorem of Casson-Gordon, Topology Appl. 125 (2002), no. 3, 571-579.

[12] K. Morimoto, Tunnel number, connected sum and meridional essential surfaces, Topology 39 (2000), no. 3, 469-485.

[13] R. Qiu and F. Lei, On the Heegaard genera of 3-manifolds containing non-separating surfaces, Topology and physics, 341-347, Nankai Tracts Math., 12, World Sci. Publ., Hackensack, NJ, 2008.

[14] M. Scharlemann, Local detection of strongly irreducible Heegaard splittings, Topology Appl. 90 (1998), no. 1-3, 135-147.

[15] M. Scharlemann and A. Thompson, Thin position for 3-manifolds, Geometric topology (Haifa, 1992), 231-238, Contemp. Math., 164, Amer. Math. Soc., Providence, RI, 1994.

[16] Heegaard splittings of (surface) $\times I$ are standard, Math. Ann. 295 (1993), no. 3, 549-564.

[17] M. Scharlemann and M. Tomova, Alternate Heegaard genus bounds distance, Geom. Topol. 10 (2006), 593-617.

[18] J. Schultens, Additivity of tunnel number for small knots, Comment. Math. Helv. 75 (2000), no. 3, 353-367.

[19] J. Schultens and R. Weidmann, Destabilizing amalgamated Heegaard splittings, Workshop on Heegaard Splittings, 319-334, Geom. Topol. Monogr., 12, Geom. Topol. Publ., Coventry, 2007.

[20] J. Souto, Distance in the curve complex and Heegaard genus, preprint.

[21] G. Yang and F. Lei, On amalgamations of Heegaard splittings with high distance, Proc. Amer. Math. Soc. 137 (2009), no. 2, 723-731.

FENGLING Li

Department of Mathematics

Harbin Institute of TeChnology

Harbin 150001, P. R. China

E-mail address: fenglingli@yahoo.com.cn 


\section{FENGCHUN LeI}

SCHOOL OF MATHEMATICS

Dalian University of TEChNOLOGY

DALIAN 116024, P. R. CHINA

E-mail address: ffcclei@yahoo.com.cn 